physical activity than others, and were capable of stimulating the whole group of ants into similar activity. Thus even when the ants are not employed in any occupation, they may still be stimulated into undirected physical activity by individuals acting as 'excitement centres'. It would be interesting to know how far this individual liveliness is correlated with hard-workingness and laziness when ants are engaged in definite tasks (see $\mathrm{Chen}^{3}$ ) and with the ability to learn (see Schneirla ${ }^{4}$ ).

20 Robert Adam Street, London, W.1. April 10.

${ }^{1}$ Wragge Morley, D., Nature, 158, 913 (1946).

${ }^{2}$ Cunliffe Barnes, T., J. Gen. Psychol., 25, 249 (1941).

${ }^{3}$ Chen, S. C., Physiol Zool., 10, 420 and 437 (1938).

- Schneirla, T. C., J. Comp. Psychol, 15, 243 (1933); 17, 303 (1934). 32,41 (1941) ; 35, 149 (1943); J. New York Ent. Soc., 52, 15 (1944), and other papers.

\section{A New Locality for Cyprinodon dispar
Rueppel}

IN the winter of 1943-44, large shoals of Cyprinodon dispar Rueppel appeared in shallow water near the shore off Tel-Aviv. In the summer of 1944 they were found to be abundant in the salt pans of Athlith, south of Haifa. Cyprinodon dispar has not hitherto been reported from the Mediterranean Sea.

The marine specimens differ distinctly in body. shape and colour from specimens from the brackish springs in the neighbourhood of the Dead Sea. A detailed description will be published elsewhere. Specimens of both populations were kept in aquaria under like conditions and bred true to type.

H. MENDELSOHN

Pedagogical Institute for Biology Tel-Aviv.

April 21.

\section{A Possible Central Mechanism for Colour Vision}

Some years ago, one of us submitted evidence to show that the conducting unit from the central area of the retina in man and the higher primates is a three-fibre unit'. It was further suggested that this anatomical fact might be related to the trichromatic theory of colour vision. Recently we have been making a detailed analysis of the main visual centre, the lateral geniculate nucleus, of the human brain, with special reference to its laminar pattern, the relative extent of the several cell layers, and their distribution over the projection areas corresponding to different parts of the retina.

In the central retinal area of the nucleus there are three cell layers related to each eye. These layers may be called $a, b$ and $c$ (the $a$ layer, which is the most superficial, being composed of much fewer and larger cells). The component fibres of the three-fibre unit ending in these layers may also be called $a, b$ and $c$ fibres. It is now possible to make the following statements with reference to the human retina and geniculate nucleus. From the central area of the retina the conducting unit is a three-fibre unit consisting of $a, b$ and $c$ fibres. At the fixation point the unit consists of $b$ and $c$ fibres; $a$ fibres are relatively very few (or may be absent altogether). From the peripheral retina there is also a functional unit of two fibres, here represented by $a$ and $(b+c)$-that is to say, one fibre of the unit terminates in layer $a$ and the other in a region of the geniculate nucleus formed by a fusion of layers $b$ and $c$. If we assume (as we believe our evidence permits us to do) that there are not great variations in the fibre-cell ratio in the geniculate nucleus, we can also say that, taking the retina as a whole, the $a$ fibres are much fewer than the $b$ or the $c$ fibres; on the other hand, the relative number of $a$ fibres increases progressively towards the periphery of the retina. Lastly, from the central area of the retina, $c$ fibres preponderate over $b$ fibres.

We are well aware of the complexities of colour vision problems, and we ourselves do not feel competent to discuss them. Nevertheless, for the consideration of those who are better qualified in that respect, we would like to put forward the following points. If it be supposed that the $a$ fibres correspond to the blue factor of trichromasy, this would explain their proportionate increase towards the periphery of the retina, which fits in with the observation that, in the distribution of colour sensitivity over the field of vision in moderate illumination, blue has the widest extent peripherally. The fact that, over the retina as a whole, the $a$ fibres are much fewer than the $b$ or $c$ fibres corresponds with the fact that, as shown in photopic visibility curves, luminosity is least in the blue part of the spectrum and reaches its maximum in the yellow part (that is, the part representing, according to the trichromatic theory, a fusion of the red and green factors). The paucity (or possible absence) of the $a$ fibres from the foveal centre accords well with the evidence that here the retina is rela. tively insensitive to blue. Lastly, it has been found ${ }^{2}$ that if monkeys are kept for some weeks in light from which the blue end of the spectrum is completely excluded, the cells in the central retinal zone of cell layer a may undergo marked atrophic changes.

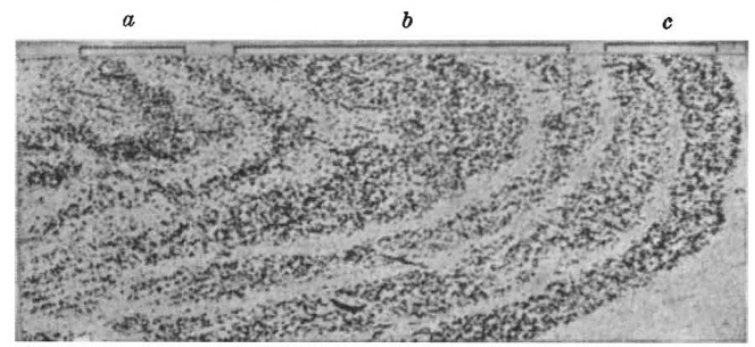

SECTION OF THE LATERAL GENICULATE BODY OF A MONKRY SHOWING SELECTIVE CELL ATROPHY IN THE CELL LAYER $a$ WHIOH REOEIVES OROSSED RETINAL FIBRES (THAT IS, THE FIRST LAYER ON THE LEFT IN THE PHOTOGRAPH). $\times 20$

In the accompanying microphotograph this selective atrophy is well shown; for reasons which are not apparent, it is mainly limited to the layer which receives crossed retinal fibres only. It should be noted, also, that some degree of cell atrophy may affect the central zones of layers $b$ and $c$, but this is less conspicuous than in layer $a$. If the $b$ and $c$ fibres are related to the red and green factors of trichromasy, then the fact that the functional conducting unit for the peripheral retina consists of $a$ and $(b+c)$ fibres explains the observation that in the field of vision the area of sensitivity for yellow extends farther than that for green or red separately; for the impulses from the receptors related to the $b$ and $c$ fibres would be conveyed to a common mass of geniculate cells (formed by the fusion in that area of layers $b$ and $c$ ) and would thus not be kept segregated in the visual paths beyond the geniculate level. 